\title{
Retos del manejo de la anticoagulación en pacientes portadores de prótesis mecánicas cardiacas con accidentes cerebrovasculares hemorrágicos
}

\author{
Ana Ruiz Rodríguez \\ Lara Ruiz Gómez

\section{Enviado: 20/06/2021} \\ Aceptado: 15/10/2021. \\ Publicado: 15/12/2021
}

Uxue Idiazabal Rodríguez

Garazi Ramírez-Escudero Ugalde

Arantza Manzanal Rey

Nora García Ibarrondo

Departamento de Cardiología. Unidad de Imagen Cardiaca. Hospital Universitario de Basurto. Bilbao. País Vasco. España

Citar como: Uxue Idiazabal Rodríguez U, Ramírez-Escudero Ugalde G, Manzanal Rey A, Ruiz Rodríguez A, Ruiz Gómez L, García Ibarrondo N. Retos del manejo de la anticoagulación en pacientes portadores de prótesis mecánicas cardiacas con accidentes cerebrovasculares hemorrágicos. RETIC. 2021 (Diciembre); 4 (3): 17-20. doi: 10.37615/retic.v4n3a5

Cite this as: Uxue Idiazabal Rodríguez U, Ramírez-Escudero Ugalde G, Manzanal Rey A, Ruiz Rodríguez A, Ruiz Gómez L, García lbarrondo N. Management of anticoagulation in patients with mechanical heart valves and hemorrhagic stroke: a clinical challenge. RETIC. 2021(December); 4 (3): 17-20. doi: 10.37615/retic.v4n3a5

\begin{tabular}{l} 
Palabras clave \\
\hline$\triangleright$ Ecocardiografía. \\
$\triangleright$ Accidente cerebrovascular \\
$\quad$ hemorrágico. \\
$\triangleright$ Prótesis mecánica cardíaca. \\
$\triangleright$ Trombo intraauricular. \\
\hline \\
Keywords \\
$\triangleright$ Echocardiography. \\
$\triangleright$ Hemorrhagic stroke. \\
$\triangleright$ Mechanical heart valve. \\
$\triangleright$ Cardiac thrombus.
\end{tabular}

\section{Presentación del caso}

Presentamos el caso de una mujer de 80 años con antecedentes de fibrilación auricular (FA) intervenida con un recambio valvular mitral por una prótesis mecánica en 1990 por una valvulopatía mitral reumática por lo que la paciente estaba anticoagulada con acenocumarol. Había presentado previamente un episodio de amaurosis fugax que se interpretó como una neuropatía óptica isquémica anterior (NOIA).

La paciente acude a Urgencias por un cuadro de afasia, asimetría facial con desviación de la comisura labial hacia la derecha y hemiplejia de extremidades derechas de tiempo de evolución indeterminado. En la tomografía computarizada se apreció (TC) un área extensa de infarto establecido asociado con un leve componente de penumbra en el territorio de la arteria cerebral media izquierda debido a una oclusión completa de la arteria carótida izquierda proximal a su porción intracraneal (Figura 1). Se suspendió la anticoagulación porque en este momento presenta un INR 4.4.

En las primeras 48 horas del ingreso tuvo un pico febril de hasta $38^{\circ} \mathrm{C}$, por lo que se extraen hemocultivos, en los que se aísla un Staphylococcus aureus meticilin resistente. En el ecocardiograma transesofágico (ETE) la prótesis mecánica mitral

\section{Correspondencia}

Uxue Idiazabal Rodríguez

Uxue_278@hotmail.com mation, and the complications she had due to her clinical situation. es normofuncionante y no hay signos de endocarditis. Sin embargo, la orejuela izquierda (OI) presenta abundante ecocontraste en su interior, lo que es muy sugestivo de un trombo incipiente (Vídeos 1, 2, 3). En el TC de control (Figura 2) se aprecia que la evolución es desfavorable con datos de transformación hemorrágica. El control de la coagulación tampoco es correcto con INR superiores a 2.5 en las analíticas realizadas por lo que se decide demorar la decisión sobre el tratamiento anticoagulante a la espera de una nueva prueba de imagen.

A pesar de que los hemocultivos de control son negativos, la paciente tiene febrícula persistente por lo que se solicita un nuevo control con ETE. En esta ocasión se aprecia una masa móvil en la aurícula izquierda compatible con un trombo libre, además de material trombótico rellenando la orejuela izquierda y presencia de ecocontraste espontáneo grado IV/IV (Video 4, Figura 3, Videos 5, 6 y 7).

La paciente se encontraba en mal estado clínico, con un déficit neurológico extenso severo (NIHSS 23), y con datos en las analíticas seriadas y en la nueva TC de control de una afectación isquémica multiorgánica, con infartos renales bilaterales y esplénico, alteración de la perfusión hepática y la persistencia de la transformación hemorrágica del infarto de la ACM izquierda con edema y efecto masa acompañantes. Tras 23 días de ingreso en los que presenta empeoramiento progresivo por fracaso multiorgánico, la paciente finalmente fallece. 
Estudio por imagen

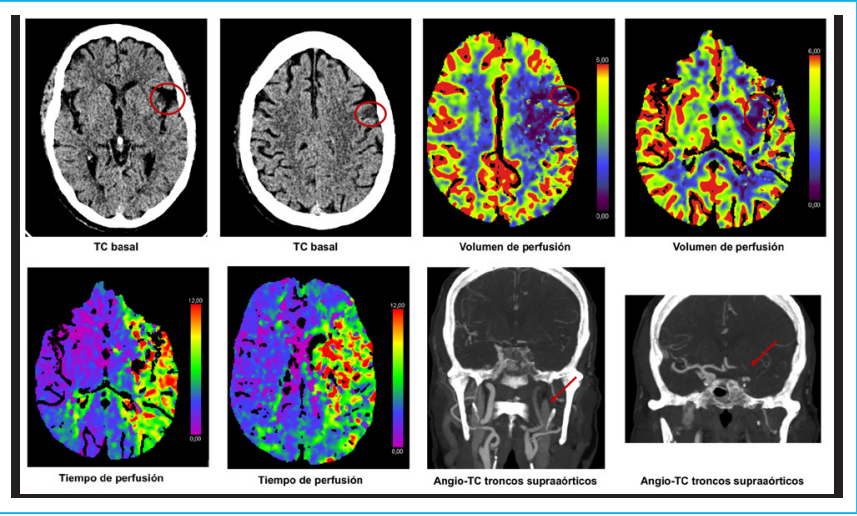

Figura 1. TC craneal en el momento del ingreso. El estudio demuestra un infarto en el territorio de la arteria cerebral media izquierda y la oclusión completa de la arteria carótida izquierda.

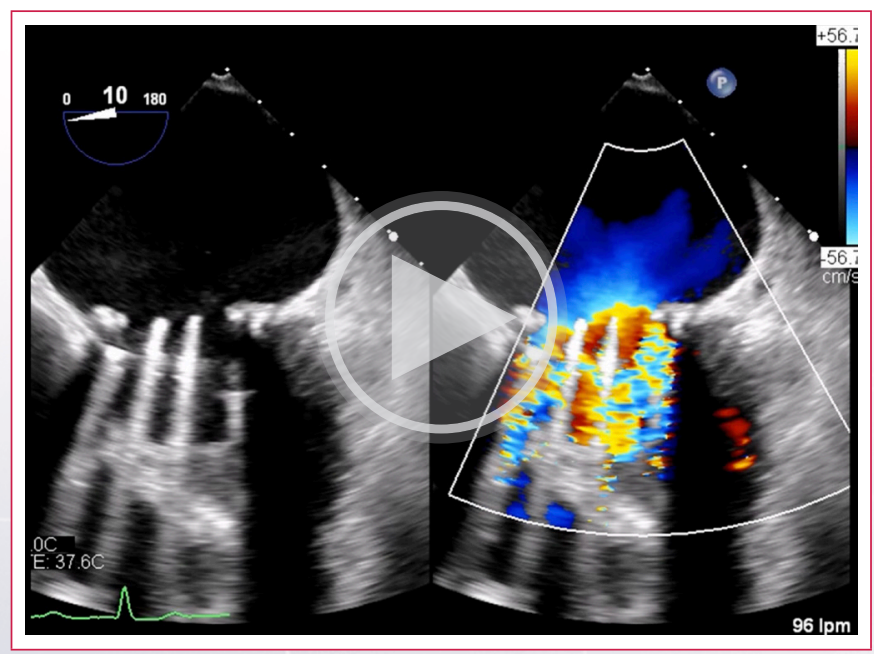

Vídeo 1. ETE basal Plano de 4 cámaras a $10^{\circ}$. Estudio de la prótesis mitral que muestra movimiento normal de los discos y jets de insuficiencia fisiológica.

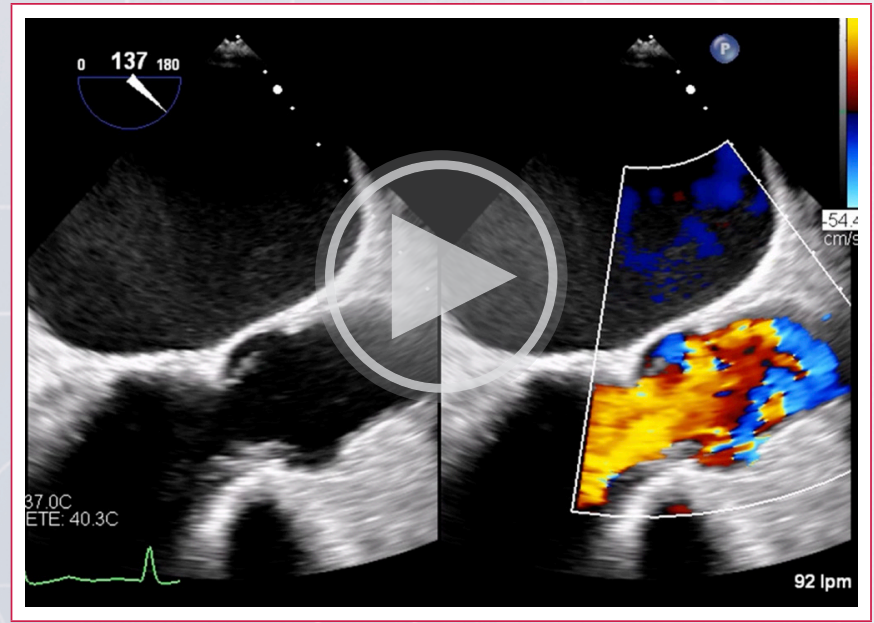

Vídeo 2. ETE basal Plano de 3 cámaras a 137. La imagen muestra una válvula aórtica anatómica y funcionalmente normal.

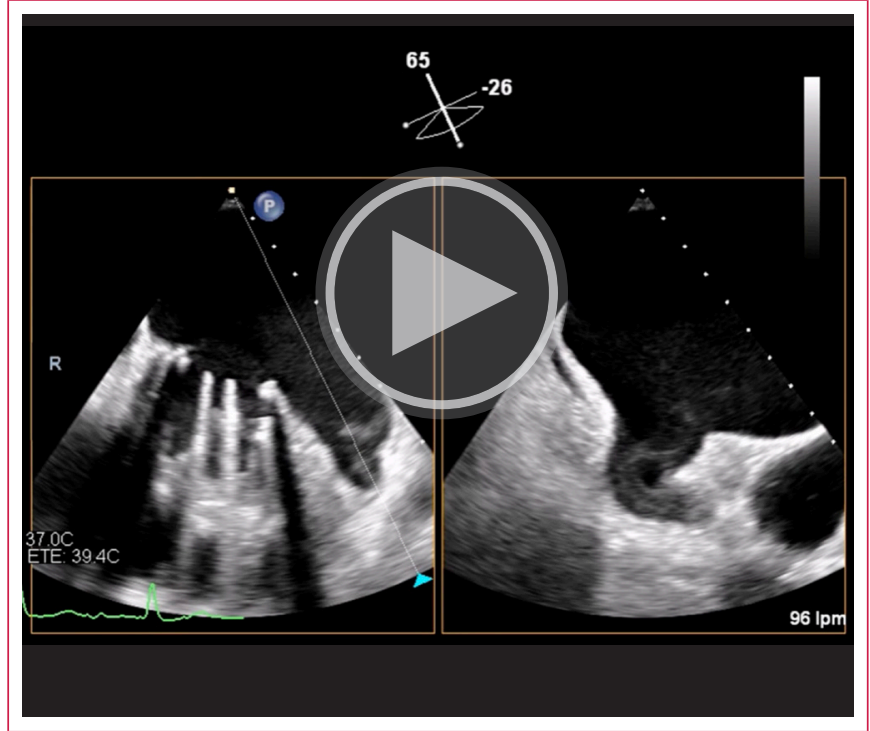

Vídeo 3. Video 1 ETE basal. Imagen biplano centrada en la orejuela izquierda que no tiene trombo, pero está rellena de ecocontraste espontáneo denso.

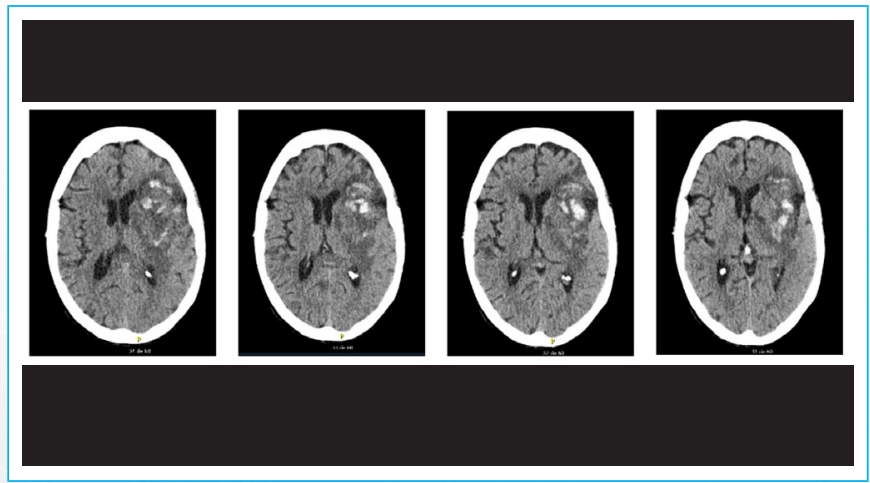

Figura 2. TC craneal en la evolución. El estudio demuestra la transformación hemorrágica del infarto asociada a efecto de masa.

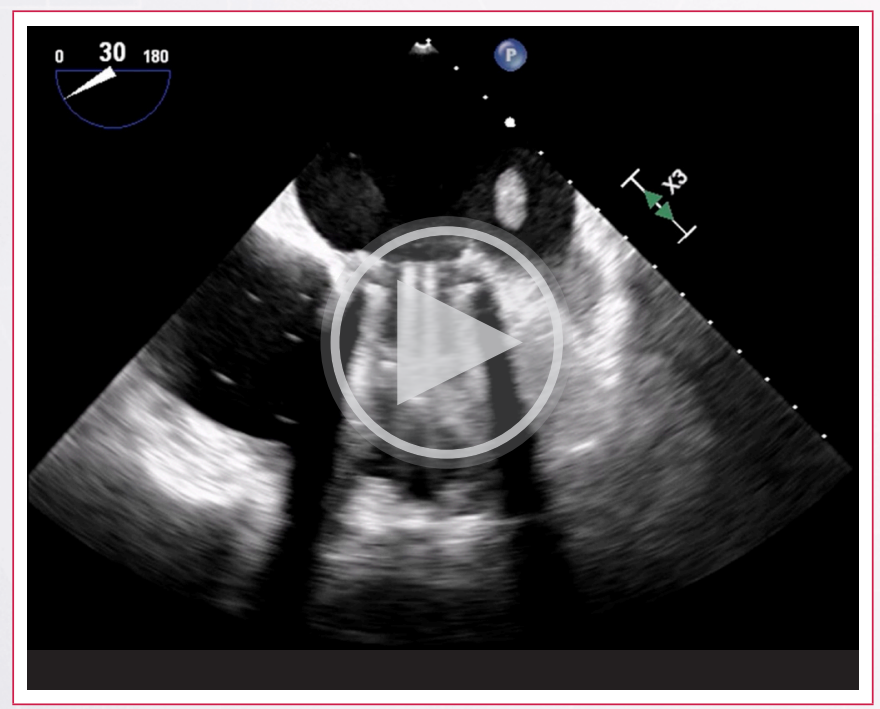

Vídeo 4. ETE de control. Plano de 4 cámaras. La prótesis mitral sigue funcionando con normalidad, pero destaca una masa móvil en la aurícula izquierda y que la orejuela izquierda ahora está tapizada por trombo. 


\section{- Casos clínicos caso 02}

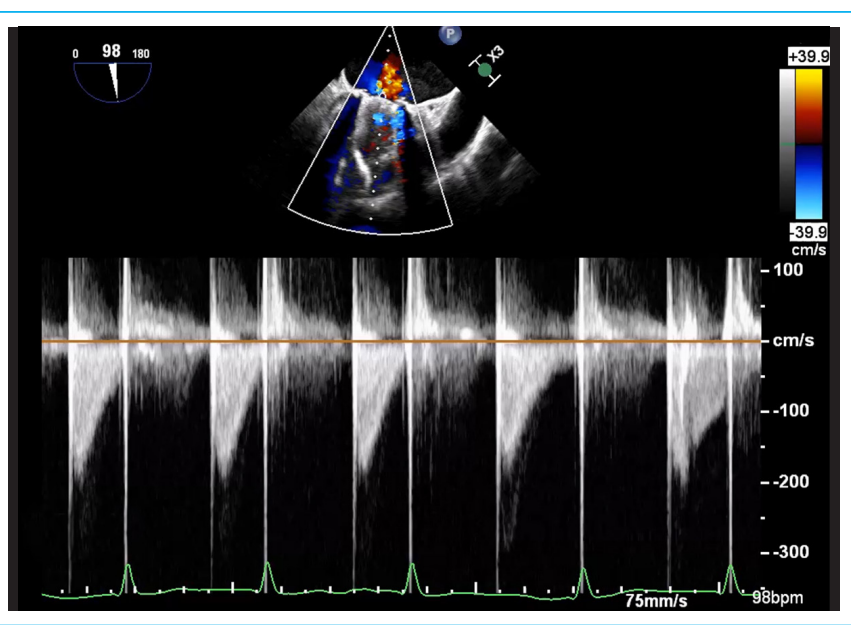

Figura 3. ETE en de control. El gradiente transvalvular mitral es normal, lo que es otro dato que indica que la prótesis funciona con normalidad.

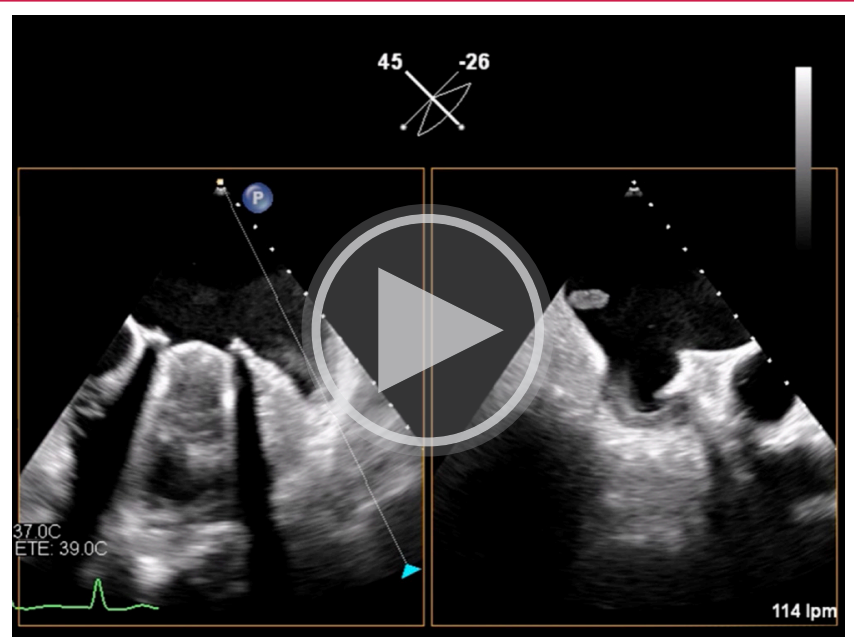

Vídeo 5. ETE de control. Imagen biplano centrado en la orejuela que muestra el cambio que se ha producido en la pared, que ahora está tapizada por trombo.

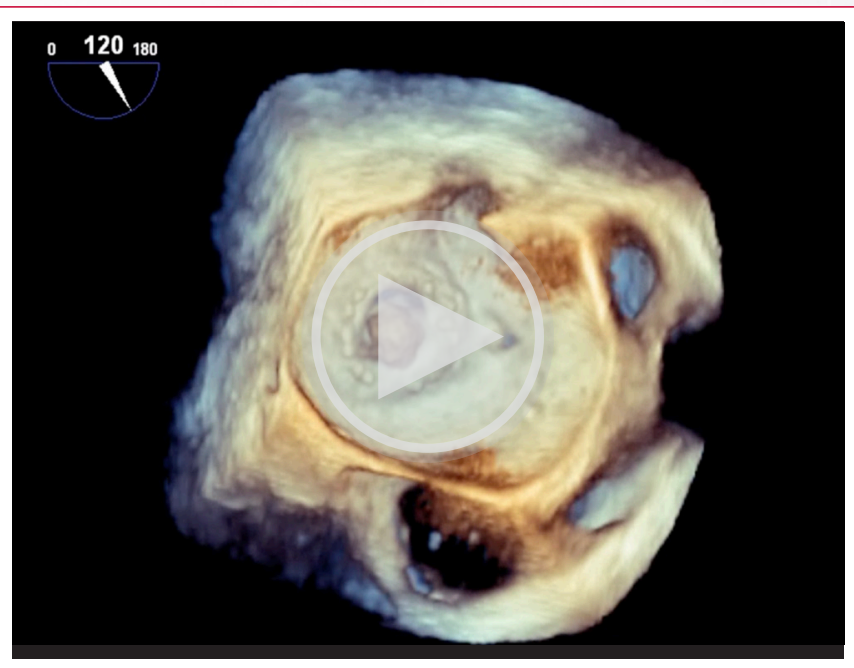

Vídeo 6. ETE de control. Imagen 3D. Destaca el trombo móvil en la aurícula izquierda y el ecocontraste en la pared.

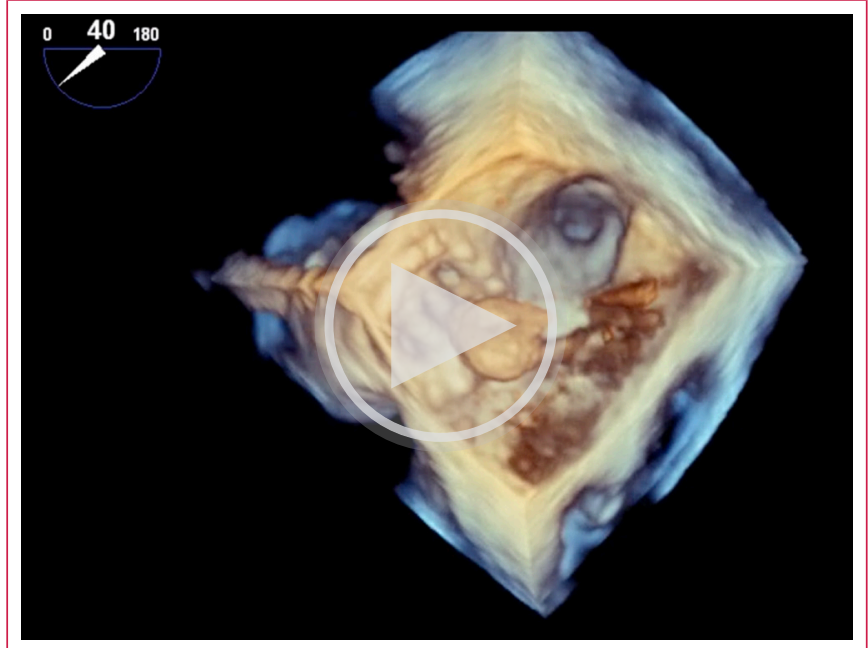

Vídeo 7. ETE de control. Imagen 3D. Esta orientación destaca el movimiento amplio de la masa en la aurícula izquierda.

\section{Discusión}

Todos los pacientes portadores de una válvula cardiaca mecánica presentan un riesgo aumentado de eventos tromboembólicos que se puede recudir mediante el uso de anticoagulantes anti-vitamina $\mathrm{K}^{1}$, pero que se asocia con un aumento del riesgo de las hemorragias intracraneales (HIC) y sus recurrencias². El valor del INR recomendado variará en función del tipo de válvula mecánica, la posición donde se implante y otros factores que puedan influir sobre el riesgo embólico'.

Para aquellos pacientes portadores de una válvula mecánica mitral con historia de un accidente cerebrovascular (ACV) isquémico o un accidente isquémico transitorio (AIT), se recomienda un INR en torno a 2.5-3.5. Además, si presentan un riesgo hemorrágico ligero, se podría añadir ácido acetil salicílico (AAS), con una dosis de 75-100 mg diarios, al tratamiento anticoagulante. Por otra parte, si a pesar de un tratamiento antitrombótico adecuado, presentasen un ACV o un embolismo sistémico, sería razonable intensificar la terapia con una dosis de $325 \mathrm{mg}$ diarios de AAS según la American Heart Association, 75-100 mg de AAS según la European Society of Cardiology ${ }^{3}$, o bien, aumentando el objetivo del valor del INR en función del riesgo hemorrágico de cada paciente

Es importante mencionar que la prevención secundaria podría ser diferente en función de si el primer episodio de ACV ha sido antes o después del recambio valvular. Sin embargo, por el momento, no existe evidencia que haga distinción entre estas dos situaciones

Uno de los escenarios clínicos más complejos es el manejo de la terapia antitrombótica en pacientes con una HIC. En aquellos pacientes con un ACV hemorrágico, la decisión de continuar con el tratamiento anticoagulante dependerá de cada escenario clínico concreto y de la indicación para el mismo. Tampoco está claro cual es el momento más adecuado para reintroducir el tratamiento anticoagulante tras una $\mathrm{HIC}^{1}$. Por este motivo, se recomienda la formación de equipos multidisciplinares formados por neurólogos, cardiólogos, radiólogos y neurocirujanos ${ }^{4}$, y valorar datos clínicos, de pruebas de imagen y los riesgos de complicaciones trombóticas como hemorrágicas a la hora de tomar la decisión ${ }^{5}$ 


\section{Conclusión}

A pesar de que todos los pacientes portadores de una válvula cardiaca mecánica requieren en teoría de tratamiento antitrombótico para reducir el riesgo de eventos tromboembólicos, el manejo de la anticoagulación en pacientes que han presentado una hemorragia intracraneal es complejo y las decisiones deben ser siempre individualizadas, valorando siempre tanto el riesgo trombótico como el hemorrágico, además del estado general de paciente.

\section{Ideas para recordar}

- El manejo de la terapia antitrombótica en pacientes con HIC supone un reto en el que hay que valorar el riesgo trombótico frente al hemorrágico.

- Se recomienda que cada caso sea evaluado de manera individualizada por equipos multidisciplinares.

\section{Bibliografía}

1. Kernan WN, Ovbiagele B, Black HR, Bravata DM, Chimowitz MI, Ezekowitz MD, Fang MC, Fisher M, Furie KL, Heck DV, Johnston SC, Kasner SE, Kittner SJ,
Mitchell PH, Rich MW, Richardson D, Schwamm LH, Wilson JA; on behalf of the American Heart Association Stroke Council, Council on Cardiovascular and Stroke Nursing, Council on Clinical Cardiology, and Council on Peripheral Vascular Disease. Guidelines for the prevention of stroke in patients with stroke and transient ischemic attack: a guideline for healthcare professionals from the American Heart Association/American Stroke Association. Stroke. 2014;45:2160-2236.

2. Hemphill JC 3rd, Greenberg SM, Anderson CS, Becker K, Bendok BR, Cushman M, Fung GL, Goldstein JN, Macdonald RL, Mitchell PH, Scott PA, Selim $\mathrm{MH}$, Woo D; on behalf of the American Heart Association Stroke Council, Council on Cardiovascular and Stroke Nursing, and Council on Clinical Cardiology. Guidelines for the management of spontaneous intracerebral hemorrhage: a guideline for healthcare professionals from the American Heart Association/American Stroke Association. Stroke. 2015;46:20322060

3. Baumgartner et al. Guía ESC/EACTS 2017 sobre el tratamiento de las valvulopatías. Rev Esp Cardiol. 2018;71(2):110.e1-e47.

4. Giakoumettis D, Alexiou GA, Vrachatis DA, Themistoklis K, Stathis P, Vavuranakis M, Themistocleous MS. Antithrombotic Treatment Management in Patients with Intracerebral Hemorrhage: Reversal and Restart. Curr Pharm Des. 2017:23(9):1392-1405. doi: 10.2174/1381612822666161205111459. PMID: 27917716

5. da Silva, I.R.F., Frontera, J.A. Resumption of Anticoagulation After Intracranial Hemorrhage. Curr Treat Options Neurol 19, 39 (2017). https://doi. org/10.1007/s11940-017-0477-y. 